\title{
Efficacy of Rifaximin Compared with Ciprofloxacin for the Treatment of Acute Infectious Diarrhea: A Randomized Controlled Multicenter Study
}

\author{
Kyoung Sup Hong*, You Sun Kim ${ }^{\dagger}$, Dong Soo $\mathrm{Han}^{\ddagger}$, Chang Hwan Choi ${ }^{\S}$, Byung-lk Jang", \\ Young-Sook Park", Kang-Moon Lee ${ }^{\#}$, Soo Teik Lee ${ }^{\star *}$, Hyun-Soo Kim ${ }^{\dagger+}$, and Joo Sung Kim* \\ Department of Internal Medicine, *Seoul National University College of Medicine, ${ }^{\dagger}$ Inje University College of Medicine, ${ }^{\dagger}$ Hanyang University \\ College of Medicine, ${ }^{s}$ Chung-Ang University College of Medicine, Seoul, "Yeungnam University College of Medicine, Daegu, "Eulji University \\ School of Medicine, Daejeon, "The Catholic University of Korea School of Medicine, Seoul, **Chonbuk National University Medical School, \\ Jeonju, and ${ }^{+\dagger}$ Chonnam National University Medical School, Gwangju, Korea
}

Background/Aims: Ciprofloxacin has been widely prescribed for acute infectious diarrhea. However, the resistance to this drug is increasing. Rifaximin is a novel but poorly absorbed rifamycin derivative. This study evaluated and compared the efficacies of rifaximin and ciprofloxacin for the treatment of acute infectious diarrhea. Methods: We performed a randomized controlled multicenter study in Korea. Patients with acute diarrhea were enrolled and randomized to receive rifaximin or ciprofloxacin for 3 days. The primary efficacy endpoint was the time to last unformed stool (TLUS). Secondary endpoints were enteric wellness (reduction of at least $50 \%$ in the number of unformed stools during 24-hour postenrollment intervals), general wellness (subjective feeling of improvement), and proportion of patients with treatment failure. Results: Intent-to-treat analysis $(n=143)$ showed no significant difference between the rifaximin and ciprofloxacin groups in the mean TLUS (36.1 hours vs 43.6 hours, $p=0.163$ ), enteric wellness $(49 \%$ vs $57 \%, p=0.428)$, general wellness $(67 \%$ vs $78 \%, p=0.189)$, or treatment failure rate $(9 \%$ vs $12 \%, p=0.841)$. The adverse events did not differ significantly between the two groups. Conclusions: These results suggest that rifaximin is as safe and effective as ciprofloxacin in the treatment of acute infectious diarrhea. (Gut Liver 2010;4:357-362)

Key Words: Acute infectious diarrhea; Rifaximin; Ciprofloxacin

\section{INTRODUCTION}

Ciprofloxacin is commonly prescribed for the treatment of moderate to severe acute infectious diarrhea including traveler's diarrhea. But, the dramatic increases in the rate of fluoroquinolones resistance have emerged since 90's in concordance with the widespread use of fluoroquinolones and now limit the usefulness of these agents in Campylobacter infections. ${ }^{1,2}$ Pathogenic Escherichia coli is known to be the most frequently identified pathogen in acute infectious diarrhea including traveler's diarrhea, ${ }^{3}$ and has become so pervasive as to account for $58 \%$ of all fecal isolates from the community acquired acute diarrheal patients during the year 2006 compared to $34 \%$ during the year 2004 in Korea. ${ }^{4}$ Moreover, nationwide surveillance of antimicrobial resistance revealed that the prevalence of fluoroquinolones-resistant $E$. coli increased steadily up to $34 \%$ of clinical isolates from the hospitals in Korea, ${ }^{5,6}$ and $15 \%$ of fecal isolates from the public health institutes which were in charge of primary care in part all over the country. ${ }^{4}$

Rifaximin is a novel, poorly absorbed rifamycin derivative for the treatment of uncomplicated traveler's diarrhea, ${ }^{7-9}$ especially caused by non-invasive pathogens, ${ }^{10}$ and was first approved in Korea in 2006. The aim of this study was to evaluate the efficacy of rifaximin compared with the ciprofloxacin for the treatment of acute in-

\footnotetext{
Correspondence to: Joo Sung Kim

Department of Internal Medicine, Seoul National University College of Medicine, 28 Yeongeon-dong, Jongno-gu, Seoul 110-744, Korea

Tel: +82-2-740-8112, Fax: +82-2-743-6701, E-mail: jooskim@snu.ac.kr
} Received on January 8, 2010. Accepted on March 29, 2010.

DOI: $10.5009 /$ gnl.2010.4.3.357 
fectious diarrhea.

\section{MATERIALS AND METHODS}

\section{Patients}

We performed a double-blind randomized controlled multicenter study in Korea from May 2008 to October 2009. The inclusion criteria were as follows: age of 19-75 years, both male and female, the presence of acute diarrhea defined as three or more unformed stools during the 24 hours preceding enrollment, accompanied by at least one of the following signs and symptoms: abdominal pain, excessive flatulence, nausea, vomiting, fecal urgency, mucus in the stool, or tenesmus. The exclusion criteria were as follows: duration of diarrhea more than 7 days, moderate or severe dehydration, fever more than $38.0^{\circ} \mathrm{C}$, blood in the stool, clinically significant disease other than diarrheal illness, pregnant or breast feeding female, any medications active against diarrheal pathogens including antibiotics and probiotics within the 7 days before randomization, more than two doses of a symptomatic antidiarrheal agents including antimotility, absorbant, or antisecretory agents within 24 hours before randomization, and any antidiarrheal agents or non-steroidal anti-inflammatory drugs or fever-reducing agents within 2 hours before randomization. This study was approved by the Institutional Review Board (IRB) of all hospitals joined the present study. All participants provided written informed consent that had been approved by the IRB. The study protocol conforms to the ethical guidelines of the 1975 Declaration of Helsinki (6th revision, 2008) as reflected in a priori approval by the IRB.

\section{Procedures}

Among acute diarrheal patients who consulted gastroenterology clinic in hospitals, consenting patients underwent screening procedures, which included the recording of medical history, a physical examination, and clinical blood studies in some cases. We accepted the double dummy method to adhere to the strict double-blind design. Eligible subjects, i.e., participants, were randomized to receive rifaximin $400 \mathrm{mg}$ plus ciprofloxacin placebo or ciprofloxacin $500 \mathrm{mg}$ plus rifaximin placebo twice a day for 3 days (day 1-3). Both placebos looked identical to the corresponding active compounds. All participants were required to complete the daily questionnaire about the time and consistency of each stool, subjective feeling of improvement, and adverse events for 5 days (day 1-5) since the administration of the study medications. Subjective feeling was recorded according to three categories: "improved", "no change or persistent", and "worsened". We communicated with participants over the telephone at day 3 to assure medication compliance and clinical response. All participants were required to revisit the clinic with the daily questionnaire filled up between day 5 and day 7 .

\section{Efficacy endpoints}

Primary efficacy endpoint was the time to last unformed stool (TLUS). Both watery and loose stool which took the shape of a container were considered as unformed stool. Secondary efficacy endpoints were the enteric wellness (defined as reduction of at least $50 \%$ in the number of unformed stools during 24-hour post-enrollment intervals compared with the 24 hours immediately preceding enrollment), the general wellness (defined as subjective feeling of improvement in symptoms during 24-hour post-enrollment intervals), and the proportion of patients who failed treatment (defined as persistence or worsening of clinical symptoms during at least 48 hours since the administration of the study medication).

\section{Randomization and sample size calculation}

We accepted blocked randomization method and generated permutationswith block size 4 or 6 at random by using the SPSS for Windows 12.0.1 (SPSS Inc., Chicago, IL, USA). Allocation number was matched to a randomization code successively by a clinical trial pharmacist of each hospital. Till completion of the study, the investigators kept the randomization table sealed off.

The sample size was based on comparison of the both treatment groups with TLUS. The null hypothesis was that rifaximin was inferior to ciprofloxacin. The non-inferiority margin was determined as 3 hours according to the expert's opinions. The assumptions for sample size calculation were as follows: standard deviation of TLUS 7 hours, alpha error 0.05 , statistical power 0.8 , drop-out rate 0.15 , and unpaired one-sided t-test. The sample size was estimated as 77 per group in consideration for dropout.

\section{Statistical analyses}

All statistical analyses were based on the intent-to-treat (ITT) principle, and the ITT population was defined as any randomized participants who took the study medications once or more. For TLUS analyses which was the primary efficacy endpoint in this study, data from patients for whom TLUS could not be calculated because of premature withdrawal caused by treatment failure or completion of the study without becoming well were censored as having a TLUS of 120 hours. Data from patients who terminated early for reasons other than treatment failure were censored at the time of the last available in- 
formation on unformed stool. Normality of data was confirmed by the 1 sample Kolmogorov-Smirnov test. Then, unpaired one-sided (non-inferiority) and two-sided (equality) t-test was performed. The non-inferiority of rifaximin to ciprofloxacin would be tested according to the lower margin of $95 \%$ confidence interval of difference of the mean TLUS (mean TLUS in rifaximin group - mean TLUS in ciprofloxacin group) between both treatment groups. If the lower limit of confidence interval was less than 3 hours which had been determined as non-inferiority margin, rifaximin would be regarded to be non-inferior to the ciprofloxacin. If the statistical power was lowered due to censored data, Cox proportional hazard models with a two-sided test and a significance level of 0.05 would be used to compare TLUS between the rifaximin and ciprofloxacin. As secondary efficacy endpoints including enteric wellness, general wellness, and treatment failure rate were all proportions, $\chi^{2}$ test was used. Fisher's exact test would be used if cell size was smaller than 5 . To adjust differences among the hospitals, Cochran-MantelHaenszel test was also used.

\section{RESULTS}

\section{Clinical characteristics of patients}

A total of 154 patients were enrolled and randomized to receive rifaximin or ciprofloxacin. Demographics and clinical characteristics were similar between both treatment groups. The pretreatment unformed stools were more frequent in the ciprofloxacin group (6.8 in the ciprofloxacin group vs 5.7 in the rifaximin group), but the difference was statistically insignificant $(p=0.057)$ (Table 1). Twenty-three participants in both treatment groups (13 in the rifaximin group, 10 in the ciprofloxacin group) were on medications including $\mathrm{H} 2$ receptor antagonist and/or proton pump inhibitor for non-ulcer dyspepsia, gastro-esophageal reflux disease (Table 1). Eleven participants who were withdrawn from the study or lost to follow-up were excluded from the efficacy analysis, and all

Table 1. Baseline Characteristics of the Patients

\begin{tabular}{|c|c|c|}
\hline & $\begin{array}{c}\text { Rifaximin } \\
(\mathrm{n}=75)\end{array}$ & $\begin{array}{l}\text { Ciprofloxacin } \\
(\mathrm{n}=68)\end{array}$ \\
\hline \multicolumn{3}{|l|}{ Age, yr } \\
\hline Mean (SD*) & $37.5(14.4)$ & $38.5(14.6)$ \\
\hline Range & $21-75$ & $19-71$ \\
\hline Sex, M/F & $43 / 32$ & $36 / 32$ \\
\hline \multicolumn{3}{|l|}{ Pretreatment unformed stools ${ }^{\dagger}$} \\
\hline $\operatorname{Mean}^{\ddagger}(\mathrm{SD})$ & $5.9(3.4)$ & $6.2(4.0)$ \\
\hline Median & 5.0 & 5.0 \\
\hline Interquartile range & 3 & 5 \\
\hline \multicolumn{3}{|l|}{ Initial symptoms, n (\%) } \\
\hline Abdominal pain & $59(79)$ & $63(93)$ \\
\hline Bloating & $32(43)$ & $16(24)$ \\
\hline Nausea/Vomiting & $26(35)$ & $29(43)$ \\
\hline Urgency & $35(47)$ & $31(46)$ \\
\hline Mucous stool & $10(13)$ & $6(9)$ \\
\hline Tenesmus & $11(15)$ & $15(22)$ \\
\hline Travel history ${ }^{\$}, \mathrm{n}(\%)$ & $13(17)$ & $8(12)$ \\
\hline \multicolumn{3}{|l|}{ Co-morbidity, n (\%) } \\
\hline Gastrointestinal diseases" & $13(17)$ & $10(15)$ \\
\hline Diabetes mellitus & $3(4)$ & $2(3)$ \\
\hline Cardiovascular diseases & $4(5)$ & $4(6)$ \\
\hline
\end{tabular}

*SD, standard deviation; ${ }^{\dagger}$ It means the counts of unformed stools in the 24-hour period before randomization; ${ }^{\ddagger}$ It was statistically insignificant $(\mathrm{p}=0.132)$; ${ }^{\mathrm{S}}$ Includes both domestic and overseas travel; "Includes non-ulcer dyspepsia, peptic ulcers, and gastroesophageal reflux disease; 'Includes hypertension, dyslipidemia, and angina.

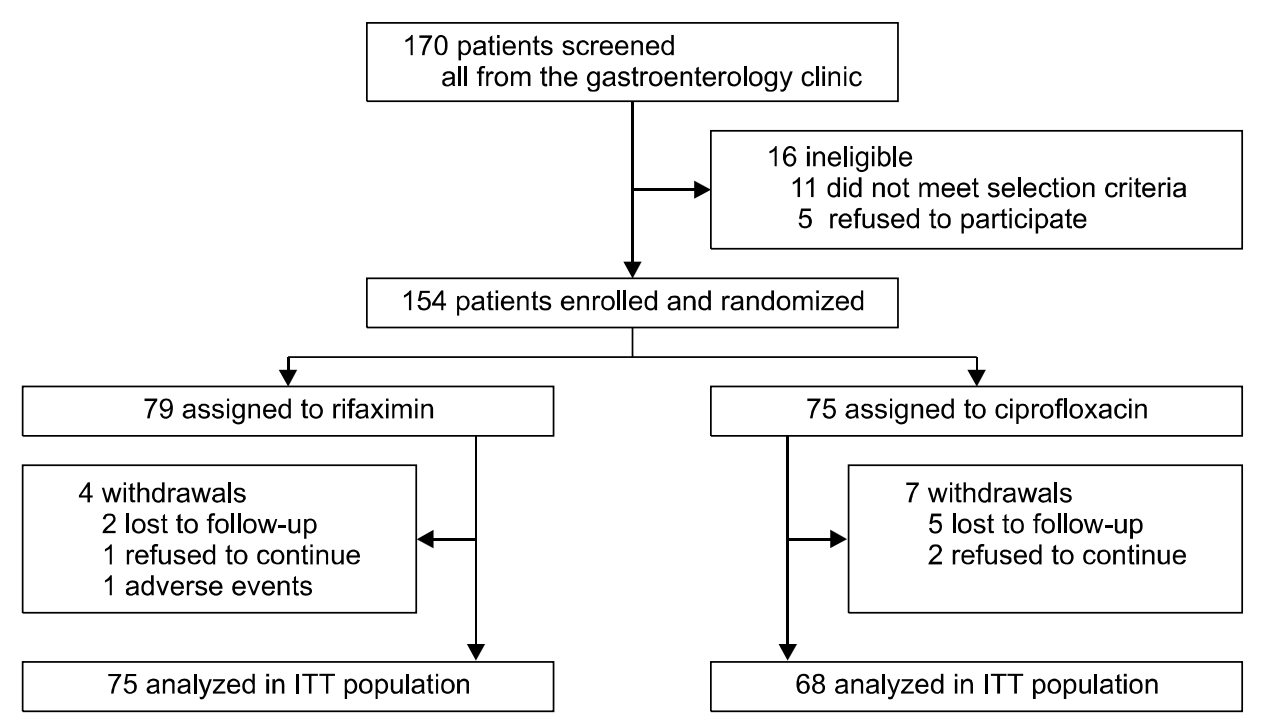

Fig. 1. Schematic flow of the study. 
the others could complete the study. The percentages of participants completing the study were similar between both groups (rifaximin, 95\% in the rifaximin group vs $91 \%$ in the ciprofloxacin group) (Fig. 1).

Compliance with both medications was good. More than $95 \%$ of participants in both groups took the prescribed number of tablets for day 1 and 2, and more than $90 \%$ took them for day 3 , as determined by daily questionnaire.

\section{Efficacy of the treatment}

Non-inferiority analysis showed the non-inferiority of rifaximin to ciprofloxacin because the lower margin of 95\% confidence interval of the difference between the TLUS of both treatment groups was -16.2 hours (unpaired one-sided $95 \%$ confidence interval, -16.2 to 1.3 hours). The mean TLUS which was the primary efficacy endpoint in this study was shorter in the rifaximin group (36.1 hours) compared with the ciprofloxacin group (43.6 hours), but the difference was not statistically significant $(p=$ 0.163). The median TLUS was similar between both treatment groups. Although the ciprofloxacin group revealed better response rates during the first 24-hour period of treatment than the rifaximin in the counts of unformed stools (enteric wellness; $57 \%$ vs $49 \%$ respectively, $\mathrm{p}=0.428$ ) and subjective symptoms (general wellness; $78 \%$ vs $67 \%$ respectively, $\mathrm{p}=0.189$ ), the difference was not statistically significant. There was no significant difference in the proportions of patients who failed in the treatment between

Table 2. Efficacy of the Treatment

\begin{tabular}{lllll}
\hline & $\begin{array}{c}\text { Rifaximin } \\
(\mathrm{n}=75)\end{array}$ & $\begin{array}{c}\text { Ciprofloxacin } \\
(\mathrm{n}=68)\end{array}$ & p-value \\
\hline TLUS* $^{*}$ & & & \\
$\quad$ Mean $\left(\mathrm{SD}^{\dagger}\right)$ & $36.1(31.3)$ & $43.6(32.2)$ & $0.163^{\ddagger}$ \\
$\quad$ Median & 34.0 & 35.0 & & \\
$\quad$ Interquartile range & 56 & 47 & & \\
Enteric wellness & & & & \\
$\quad-24 \mathrm{hr}$ & $37(49 \%)$ & $39(57 \%)$ & $0.428^{\|}(0.252)$ \\
$-48 \mathrm{hr}$ & $62(83 \%)$ & $55(81 \%)$ & $0.953(0.799)$ \\
General wellness & & & & \\
$-24 \mathrm{hr}$ & $50(67 \%)$ & $53(78 \%)$ & $0.189(0.140)$ \\
$-48 \mathrm{hr}$ & $65(87 \%)$ & $57(84 \%)$ & $0.808(0.619)$ \\
Treatment failure** & $7(9 \%)$ & $8(12 \%)$ & $0.841(0.618)$ \\
\hline
\end{tabular}

${ }^{*}$ TLUS, the time to last unformed stool; ${ }^{\dagger} \mathrm{SD}$, standard deviation; ${ }^{f}$ Determined by unpaired two-sided t-test; ${ }^{\S}$ Reduction of at least $50 \%$ in the number of unformed stools; "Determined by $\chi^{2}$ test; " Determined by Cochran-Mantel-Haenszel test by hospital strata; ${ }^{\#}$ Subjective feeling of improvement in symptoms; **Persistence or worsening of clinical symptoms during at least 48 hours since the administration of the study medication. both treatment groups $(\mathrm{p}=0.841)$. Because all of 15 participants who failed treatment could be followed up and became well within 5-day period of the study, there were no need of data censoring as having a TLUS of 120 hours. Consequently, the primary and secondary efficacy endpoints were all similar between both rifaximin and ciprofloxacin groups (Table 2).

\section{Adverse events of the treatment}

The incidence of adverse events was similar between both rifaximin and ciprofloxacin groups. One participant who had underlying diabetes mellitus in the rifaximin group withdrew prematurely from the study because of serious adverse events (hypoglycemia after the ingestion of oral anti-diabetic agent during fasting) that were considered by the investigator to be not drug-related (Table 3). No participant in both rifaximin and ciprofloxacin group withdrew prematurely because of drug-related adverse events, i.e., adverse drug reaction.

\section{DISCUSSION}

Infectious or noninfectious causes may be responsible for acute diarrhea. Noninfectious causes of diarrhea include drugs, food allergies, inflammatory bowel disease, and other states such as thyrotoxicosis and the carcinoid syndrome, which become more common as the course of the diarrhea persists and becomes chronic. Because we excluded patients whose duration of diarrhea was more than 7 days and all participants including 15 participants who failed treatment became well within 5-day period of the study, the participants of this study could be regarded to catch acute infectious diarrhea.

In this study, rifaximin showed comparable efficacy with ciprofloxacin in patients with acute infectious diar-

Table 3. Adverse Events of the Treatment

\begin{tabular}{|c|c|c|}
\hline & $\begin{array}{c}\text { Rifaximin } \\
(\mathrm{n}=79)\end{array}$ & $\begin{array}{l}\text { Ciprofloxacin } \\
\quad(\mathrm{n}=75)\end{array}$ \\
\hline Adverse events & $5\left(5^{*}\right)$ & $9\left(7^{*}\right)$ \\
\hline Headache & 1 & 3 \\
\hline Nausea & 1 & 2 \\
\hline Febrile sense & 1 & 1 \\
\hline Abdominal pain & 1 & 3 \\
\hline Serious $\mathrm{AEs}^{\dagger}$ & $1^{\ddagger}$ & 0 \\
\hline
\end{tabular}

*Numberof the participants who reported any adverse event; ${ }^{\dagger}$ Any adverse events that result in death, life-threatening, prolonged hospitalization, or irreversible disability; ${ }^{\dagger}$ Hypoglycemic event in a DM patient who ingested oral anti-diabetic agents during fasting. 
rhea without fever or bloody stool. However, in contrary to the previous studies which reported same or better overall efficacy of the ciprofloxacin, ${ }^{8,10}$ the rifaximin group revealed shorter mean TLUS than that in the ciprofloxacin group in this study although the differences were statistically insignificant. Moreover, although we applied more strict criteria to determine "treatment failure" compared with the previous studies in which the presence of residual symptoms after at least 24 hours of therapy was regarded as failed treatment, ${ }^{8,10}$ more patients up to $12 \%$ of all participants who were randomized into the ciprofloxacin group were determined as "treatment failure" in this study compared with $6-7 \%$ in the previous studies. ${ }^{8,10}$ This result may be caused by the increased ciprofloxacin resistance of $E$. coli which is known as the dominant pathogen in adult patients with acute infectious diarrhea. Quinolone-resistant E. coli (QREC) strains have been being isolated with increasing frequency from the community and hospital since $90 \mathrm{~s},{ }^{11,12}$ and up to $38.6 \%$ of clinical isolates collected from January 2008 until December 2008 was found as QREC in Seoul National University Hospital in Korea. ${ }^{13}$ It is important to note that the isolates of QREC revealed little evidence of clonal spread and might have emerged in direct response to the selective pressure exerted by prior fluoroquinolones use through the analysis on genetic diversity of clinical isolates from one teaching hospital in Korea. ${ }^{14}$ Moreover, through a case-control study it was reported that recent fluoroquinolones use, residence in a long-term care facility, recent aminoglycoside use, and older age were all noted to be independent risk factors for fluoroquinolones resistance among patients with nosocomial $E$. coli infections through a casecontrol study. ${ }^{15}$ In contrary to the two previous studies for which only travelers visited at least one developing country had been enrolled, we recruited all the participants from gastroenterology clinics of 9 hospitals and some of the participants had co-morbidities including gastro-esophageal reflux disease, diabetes mellitus and hypertension etc, although all these were mild and well-controlled (Table 1). As expected, the mean age of participants in this study was 38 years, which was 5-12 years higher than those in the previous studies. ${ }^{8,10}$ Therefore, it seems reasonable that the increased prevalence of QREC resulted in the decreased efficacy of ciprofloxacin for the treatment of acute infectious diarrhea in this study.

Rifaximin has a broad spectrum of antimicrobial action, covering Gram-positive and Gram-negative, both aerobes and anaerobes. ${ }^{16}$ It was first approved in Italy in 1987, ${ }^{9}$ was approved by the US Food and Drug Administration in 2004 for the treatment of uncomplicated traveler's diarrhea. ${ }^{17}$ Rifaximin's additional pyridoimidazole ring makes it virtually nonabsorbable, ${ }^{18}$ enabling it to achieve the extremely high concentration up to the range of $4,000-8,000 \mu \mathrm{g} / \mathrm{g}$ stool in the gastrointestinal tract and to be active against enteric infection. ${ }^{19}$ One concern with a broad spectrum antibiotics is the risk of depleting normal gut flora. However, there was only a minor change in bacterial counts which reverted back to pre-treatment values during the washout period despite the high dose (i.e., $1,800 \mathrm{mg}$ daily) of rifaximin. ${ }^{20}$ Another concern, actually with all antimicrobial agents, is the potential to induce antibacterial resistance. Reportedly, rifaximin resistance occurred in the gut flora of healthy volunteers who received treatment with rifaximin at dose of $800 \mathrm{mg} /$ day for 5 days, but the resistance rates decreased to less than $20 \%$ of the intestinal flora within 2 weeks after the end of rifaximin treatment. ${ }^{21}$ Further studies are warranted to investigate the precise mechanism and clinical significance of such a phenomenon, which must be preceded to determine its possibility of a potential substitute for ciprofloxacin.

Even if the absence of microbiologic analysis for infectious organism was the weak point in this study, we performed the multicenter study to evaluate the efficacy of rifaximin and ciprofloxacin in acute diarrheal patients under the situation of highly prevalent QREC, especially in referral hospitals in Korea. As a result, comparable efficacy of both treatments was shown, and the decreased efficacy of the ciprofloxacin was observed apparently compared with those from the previous studies. ${ }^{8,10}$ According to the Infectious Diseases Society of America guidelines for the management of acute infectious diarrhea, two situations in which empirical antibiotics are commonly recommended without obtaining a fecal specimen are the moderate to severe cases of traveler's diarrhea and febrile dysenteric illness, especially those believed to have moderate to severe invasive disease, and fluoroquinolones are recommended as first choice in adults patients. ${ }^{22}$ Nevertheless, it is frequently inevitable in daily practice to prescribe empirical antibiotics for the treatment of acute diarrheal patients who are not obviously categorized into the two situations, especially to prevent dehydration in elderly patients with co-morbidities.

In conclusion, rifaximin is as safe and effective as the ciprofloxacin in the patients with acute infectious diarrhea, and further studies are needed to update the current recommendation of antibiotic regimen against moderate to severe acute infectious diarrhea.

\section{ACKNOWLEDGEMENTS}

This study was supported by the research fund of the 
Korean Association for the Study of Intestinal Diseases.

\section{REFERENCES}

1. Smith KE, Besser JM, Hedberg CW, et al. Quinolone-resistant Campylobacter jejuni infections in Minnesota, 1992-1998. Investigation Team. N Engl J Med 1999;340: 1525-1532.

2. Yang JR, Wu HS, Chiang CS, Mu JJ. Pediatric campylobacteriosis in northern Taiwan from 2003 to 2005. BMC Infect Dis 2008;8:151.

3. Steffen R, Collard F, Tornieporth N, et al. Epidemiology, etiology, and impact of traveler's diarrhea in Jamaica. JAMA 1999;281:811-817.

4. Cho SH, Shin HH, Choi YH, Park MS, Lee BK. Enteric bacteria isolated from acute diarrheal patients in the Republic of Korea between the year 2004 and 2006. J Microbiol 2008;46:325-330.

5. Chong Y, Lee K, Park YJ, et al. Korean Nationwide Surveillance of Antimicrobial Resistance of bacteria in 1997. Yonsei Med J 1998;39:569-577.

6. Lee K, Kim YA, Park YJ, et al. Increasing prevalence of vancomycin-resistant enterococci, and cefoxitin-, imipenem- and fluoroquinolone-resistant gram-negative bacilli: a KONSAR study in 2002. Yonsei Med J 2004;45:598-608.

7. Descombe JJ, Dubourg D, Picard M, Palazzini E. Pharmacokinetic study of rifaximin after oral administration in healthy volunteers. Int J Clin Pharmacol Res 1994;14:51-56.

8. DuPont HL, Jiang ZD, Ericsson CD, et al. Rifaximin versus ciprofloxacin for the treatment of traveler's diarrhea: a randomized, double-blind clinical trial. Clin Infect Dis 2001;33:1807-1815.

9. Adachi JA, DuPont HL. Rifaximin: a novel nonabsorbed rifamycin for gastrointestinal disorders. Clin Infect Dis 2006;42:541-547.

10. Taylor DN, Bourgeois AL, Ericsson CD, et al. A randomized, double-blind, multicenter study of rifaximin compared with placebo and with ciprofloxacin in the treatment of travelers' diarrhea. Am J Trop Med Hyg 2006;74: 1060-1066.

11. Peña C, Albareda JM, Pallares R, Pujol M, Tubau F, Ariza
J. Relationship between quinolone use and emergence of ciprofloxacin-resistant Escherichia coli in bloodstream infections. Antimicrob Agents Chemother 1995;39:520-524.

12. Ena J, López-Perezagua MM, Martínez-Peinado C, Cia-Barrio MA, Ruíz-López I. Emergence of ciprofloxacin resistance in Escherichia coli isolates after widespread use of fluoroquinolones. Diagn Microbiol Infect Dis 1998;30:103-107.

13. Kim EC. The analysis of antibiotics resistance 2008. Seoul National University Hospital Infection Control Newsletter 2009;15:5-12.

14. Cheong HJ, Yoo CW, Sohn JW, Kim WJ, Kim MJ, Park SC. Bacteremia due to quinolone-resistant Escherichia coli in a teaching hospital in South Korea. Clin Infect Dis 2001;33: 48-53.

15. Lautenbach E, Fishman NO, Bilker WB, et al. Risk factors for fluoroquinolone resistance in nosocomial Escherichia coli and Klebsiella pneumoniae infections. Arch Intern Med 2002;162:2469-2477.

16. Robins GW, Wellington K. Rifaximin: a review of its use in the management of traveller's diarrhoea. Drugs 2005; 65:1697-1713.

17. Salix Pharmaceuticals. Xifaxan (rifaximin) tablets [prescribing information]. Palo Alto: Salix Pharmaceuticals, 2004.

18. Martini S, Bonechi C, Corbini G, Donati A, Rossi C. Solution structure of rifaximin and its synthetic derivative rifaximin OR determined by experimental NMR and theoretical simulation methods. Bioorg Med Chem 2004;12: 2163-2172.

19. Jiang ZD, Ke S, Palazzini E, Riopel L, Dupont H. In vitro activity and fecal concentration of rifaximin after oral administration. Antimicrob Agents Chemother 2000;44: 2205-2206.

20. Brigidi P, Swennen E, Rizzello F, Bozzolasco M, Matteuzzi D. Effects of rifaximin administration on the intestinal microbiota in patients with ulcerative colitis. J Chemother 2002;14:290-295.

21. De Leo C, Eftimiadi C, Schito GC. Rapid disappearance from the intestinal tract of bacteria resistant to rifaximin. Drugs Exp Clin Res 1986;12:979-981.

22. Guerrant RL, Van Gilder T, Steiner TS, et al. Practice guidelines for the management of infectious diarrhea. Clin Infect Dis 2001;32:331-351. 